\title{
Impact de la variété et de la maturité à la récolte sur l'effet de la chloration et de l'emballage du macabo (Xanthosoma spp.) en entreposage
}

\author{
René Mbonomo BIKOMO ${ }^{1 *}$ et AMOUGOU AKOA ${ }^{2}$ \\ ${ }^{I}$ Département d'Agriculture, Faculté d'Agronomie et des Sciences Agricoles, \\ Université de Dschang, BP 222 Dschang, Cameroun. \\ ${ }^{2}$ Chef de Département de Biologie et Physiologie Végétales, Faculté de Sciences, \\ Université de Yaoundé I, BP. 812 Yaoundé, Cameroun. \\ * Auteur correspondant, E-mail: r_bikomo@yahoo.fr, Tel (237) 77652969.
}

\section{RESUME}

Les tubercules de deux variétés de macabo (Xanthosoma sagittifolium), le rouge et le blanc, étaient conditionnés et testés dans un contexte factoriel post récolte pour améliorer la performance à l'entreposage. La récolte du macabo après 10 ou 11 mois a induit une amélioration de la qualité globale et l'aptitude des tubercules à un entreposage prolongé. L'utilisation de l'emballage en sacs plastiques perforés a de façon significative réduit la perte de poids, à l'opposé des sacs de jute ou de l'absence d'emballage. La solution de chlore $(1 \% \mathrm{NaOCl})$, utilisée pour trempage bactéricide n'a pas neutralisé la pourriture. Le macabo blanc a développé le bourgeonnement le plus abondant, tandis que la variété rouge a accusé une perte en eau continue et plus prononcée en absence d'emballage et pour les tubercules récoltés avant 10 mois de maturité. La palatabilité du macabo était restée sans influence du chlore et la flaveur du macabo récolté à 10 et 11 mois était plus appréciée.

(C) 2009 International Formulae Group. All rights reserved.

Mots clés : Tubercules, maturation, conditionnement, chlore, stockage, qualité.

\section{INTRODUCTION}

La détérioration post récolte du macabo est causée par des réactions physiologiques endogènes ou des attaques d'agents pathogènes pouvant dans les deux cas être aggravés par des défectuosités physiques (Booth, 1974; Ogundana, 1977; Coursey, 1983; Nzietchueng, 1983). En fait, le macabo se caractérise également par sa susceptibilité à l'endommagement résultant des blessures, des entailles et des lésions de pellicule pendant la récolte (O’Hair et Asokan, 1986) et des meurtrissures durant le transport (Burton, 1982; Bikomo, 1988).

La détérioration du macabo entreposé peut provenir du stade immature des tissus des tubercules à leur récolte (Ora, 1977).
Le mésocarpe fibreux, séché et humidifié, mais non mouillé du fruit de cocotier, constitue un milieu d'entreposage satisfaisant du macabo (Passam, 1982). De la sciure de bois dans des caisses ou des sacs en polyéthylène a favorisé la préservation d'une qualité acceptable des racines de manioc pendant 8 à 4 semaines respectivement (Rickard et Coursey, 1981). De même des sacs en plastique perforé ont maintenu une apparence fraîche et ferme de la banane (Shillingford, 1978) et du macabo (Bikomo, 1988).

L'objectif général de cette étude est de montrer l'influence de la variété et de la maturité à la récolte sur l'effet de la chloration et de l'emballage du macabo. Les objectifs spécifiques portent sur l'identification de 
l'effet combiné des facteurs étudiés sur le comportement des tubercules de macabo entreposés, notamment: la variation de la présentation physique globale, la variation de certaines caractéristiques physico-chimiques, la variation de la susceptibilité aux attaques d'agents pathologiques, l'évolution de la qualité organoleptique.

\section{MATERIEL ET METHODES}

Des tubercules frais de deux variétés sélectionnées de macabo (Xanthosoma sagittifolium L.), la variété à chair rouge et celle à chair blanche étaient récoltés au sud du Cameroun et étaient acheminés sans lavage préalable dans des sacs de jute transportés par un véhicule pick up, pour stockage à Dschang. Ces produits étaient regroupés suivant la variété, et mélangés au sein de la même variété. Les tubercules étaient par la suite triés sur la base d'absence de défectuosités (fragmentation, lésions de pellicule, meurtrissures et pourriture) et étaient calibrés et organisés de manière aléatoire en échantillons de 10 tubercules, pour une durée d'entreposage de 3 mois.

Les facteurs étudiés, la variété, le stade de maturité, la chloration et l'emballage ont constitué des traitements en distribution factorielle dans un dispositif expérimental en blocs complètement randomisés à 3 répétitions, chaque variété constituant un bloc. Les différents seuils de maturité retenus étaient 8, 9, 10 et 11 mois. La solution de chlore préparée pour trempage des tubercules était obtenue par dilution de l'hypochlorite de sodium commercialisé, dans de l'eau, à la dose de 1\% (Bikomo et Brecht, 1991). Les deux types d'emballage étaient des sacs en jute et en plastique courants. Ceux en plastique étaient perforés de trous de $8 \mathrm{~mm}$ de diamètre et suivant un écartement de $10 \mathrm{~cm}$.

Des traitements combinés de tous les niveaux des facteurs étudiés étaient administrés de manière aléatoire sur les différents échantillons. Les tubercules témoins étaient disposés en une seule couche sur les rayons de l'étagère grillagée d'entreposage, tandis que les sacs en jute et ceux en plastique étaient fermés par rabattement simple du bord d'ouverture après introduction des tubercules et avant leur disposition dans l'étagère de stockage, placée sous conditions ambiantes moyennes de température de $21{ }^{\circ} \mathrm{C}$ et d'humidité relative de $76,8 \%$ prédominant à Dschang.

L'appréciation hebdomadaire de la qualité des tubercules entreposés prenait en compte la présentation physique globale visible, la perte de poids, l'incidence de la pourriture et le bourgeonnement. De plus, une évaluation mensuelle était réalisée, sur la variation de la teneur en eau et de la teneur en matière sèche de tubercules. La mesure du poids au gramme près des échantillons était effectuée au moyen d'une balance électronique Fisher Scientific, Model XT3000 DR de grande précision. L'évaluation du bourgeonnement était faite par la mesure de la longueur totale des bourgeons développés sur les tubercules. La teneur en eau et la teneur en matière sèche étaient quantifiées par pesée des échantillons après refroidissement dans un dessiccateur et broyage (broyeur Osterizer, Model 548 41A) et suivant un séjour dans une étuve à circulation d'air chaud Fisher Scientific, Model $738 \mathrm{~F}$, à $75^{\circ} \mathrm{C}$ pendant 24 heures (Bainbridge et al., 1996). Cette étape était suivie d'une seconde pesée de ces échantillons avec une balance analytique Mettler, Model AE 200.

L'apparence physique globale et l'incidence de pourriture étaient évaluées avec référence à la méthode d'analyse descriptive (Kadel et al., 1973; Larmond, 1977; Bikomo, 1988), comportant les seuils de qualité suivants: absence de défectuosités (pourriture, bourgeons, durcissement, flétrissement, état sain) $=9$, très bon état, défectuosités légères $=8$, bon état, défectuosités déjà visibles $=7$, assez bon état, devenant indésirables $=6$, acceptable, défectuosités indésirables, compromettant la qualité commerciale $=5$, assez mauvais état, seuil minimum de qualité commercialisable $=4$, non commercialisable, mauvais état $=3$, très mauvais état, nécessité d'élimination des parties détériorées avant utilisation $=2$, mauvais état extrême, non utilisable $=1$.

Un panel de dégustateurs amateurs âgés de 20 à 50 ans a apprécié la flaveur du macabo cuit par ébullition à l'eau, selon la méthode d'analyse descriptive (Larmond, 1977; Watts et al., 1991; Tiky, 1998; Emmanuel-Ikpeme et al., 2007), se présentant tels que suit: préférence effective $=5$, préférence modérée $=4$, neutralité $=3$, rejet modéré $=2$, rejet total $=1$. 
A cet effet, des échantillons de macabo cubiques de $1,5 \mathrm{~cm}$ de côté étaient distribués aux évaluateurs qui, après mastication et dégustation, les crachaient, puis se rinçaient la bouche avec l'eau de robinet entre les dégustations.

Une transformation angulaire des données originales, exprimées en pourcentages, était effectuée. L'analyse statistique des données ainsi transformées était assurée selon la méthode MSTAT, avec le test Fisher à $5 \%$ et la méthode de Duncan pour la séparation des moyennes lorsque les différences significatives étaient détectées entre les effets des traitements.

\section{RESULTATS}

Au terme de 3 mois d'entreposage, des différences statistiquement significatives $(\mathrm{p}<0,05)$ étaient observées sur la qualité des tubercules stockés, résultant de l'influence combinée des traitements testés (Tableau 1). Ces différences étaient nettement remarquables sur l'apparence physique globale, la perte de poids, l'incidence de la pourriture et le bourgeonnement.

La variété du macabo a causé une incidence significative $(\mathrm{p}<0,05)$ sur la perte de poids au cours de l'entreposage des tubercules (Tableau 1). Le macabo blanc a le moins perdu du poids, en comparaison de la réduction de poids considérable enregistrée sur le macabo rouge pendant la même phase.

Le macabo à chair blanche a manifesté une forte tendance à bourgeonner tandis que ce phénomène était moindre ou inexistant sur le macabo rouge de même stade de maturité (Tableau 2).

Le stage de maturité a produit un impact significatif $\quad(p<0,05) \quad$ dans l'amélioration de l'apparence physique des tubercules. La récolte des tubercules à 10 et 11 mois a de manière remarquable améliorée la présentation visuelle des tubercules entreposés (Tableau 2), notamment dans leur fraîcheur et leur turgescence. Au contraire, les tubercules immatures, en particulier ceux de la variété rouge du macabo, ont développé un durcissement accompagné d'un flétrissement et d'une décoloration indésirables.

Une amélioration significative $(\mathrm{p}<0,05)$ était enregistrée sur du macabo traité avec l'hypochlorite de sodium à la dose de $1 \%$ et sur des tubercules récoltés à 10 ou 11 mois, indépendamment de la variété (Tableau 1).

Tableau 1: Analyse de la variance de l'effet de la variété, du stade de maturité, de la chloration et de l'emballage sur les caractéristiques suivants du macabo entreposé : La présentation physique globale (PPG), l'incidence de la pourriture (IP), la perte de poids (PP) et le bourgeonnement $(\mathrm{Bo})$ et la palatabilité $(\mathrm{Pa})$.

\begin{tabular}{lccccc}
\hline \multirow{2}{*}{ Source de variation } & \multicolumn{5}{c}{ Carrés moyens } \\
& dl & PPG & IP & PP & Bo \\
\hline Var & 1 & $26,43^{\text {ns }}$ & $697,88^{\text {ns }}$ & $2528,49^{* *}$ & $2258,63^{* *}$ \\
Mat & 3 & $1315,59^{* *}$ & $315,35^{\text {ns }}$ & $1300,76^{\text {xx }}$ & $18,39^{\text {xx }}$ \\
Var x Mat & 3 & $94,53^{* *}$ & $1820,56^{* *}$ & $11,53^{\text {ns }}$ & $21,24^{* *}$ \\
Chl & 1 & $1824,36^{* *}$ & $663,45^{\text {ns }}$ & $136,83^{* *}$ & $11,17^{* \text { ns }}$ \\
Var x Chl & 1 & $586,29^{* *}$ & $298,28^{\text {ns }}$ & $4,93^{\text {ns }}$ & $1,42^{\text {ns }}$ \\
Mat x Chl & 3 & $630,66^{* *}$ & $390,67^{\text {ns }}$ & $10,59^{\text {ns }}$ & $23,39^{* *}$ \\
Var x Mat x Chrl & 3 & $345,07^{* *}$ & $159,31^{\text {ns }}$ & $69,41^{* *}$ & $17,84^{* *}$ \\
Emb & 2 & $79,67^{\text {ns }}$ & $459,50^{\text {ns }}$ & $104,41^{* *}$ & $24,78^{\text {ns }}$ \\
Var x Emb & 2 & $235,42^{* *}$ & $22,98^{\text {ns }}$ & $19,17^{\text {ns }}$ & $10,62^{\text {ns }}$ \\
Mat x Emb & 6 & $99,77^{\text {ns }}$ & $348,50^{\text {ns }}$ & $14,77^{\text {ns }}$ & $22,32^{* *}$ \\
Var x Mat x Emb & 6 & $209,69^{* *}$ & $444,75^{* *}$ & $49,47^{* *}$ & $20,61^{* *}$ \\
Chl x Emb & 2 & $114,65^{\text {ns }}$ & $21,38^{\text {ns }}$ & $21,35^{\text {ns }}$ & $14,75^{\text {ns }}$ \\
Var x Chl & 2 & $1,59^{\text {ns }}$ & $766,60^{* *}$ & $15,24^{\text {ns }}$ & $7,68^{\text {ns }}$ \\
Var x Chl x Emb & 6 & $68,43^{\text {ns }}$ & $103,67^{\text {ns }}$ & $6,77^{\text {ns }}$ & $4,94^{\text {ns }}$ \\
Mat x Chl x Emb & 6 & 94,01 & 94,64 & 2,09 & 3,55 \\
Erreur & 96 & 66,79 & 185,70 & 10,43 & 5,62 \\
\hline ns test non significatif à $\alpha=0,05 ;{ }^{* *}=$ test significatif à $\alpha=0,05 ;$ Var $=$ variété $;$ Mat $=$ stade de maturité $;$ \\
Chl = chloration ; Emb = emballage.
\end{tabular}


Tableau 2 : Effet de la variété, du degré de maturité, de la chloration et de l'emballage sur les caractéristiques physiques et physiologiques, et la qualité organoleptique du macabo entreposé suivantes : La présentation physique globale (PPG), perte de poids (PP), bourgeonnement (Bo) et palatabilité $(\mathrm{Pa})$.

\begin{tabular}{|c|c|c|c|c|}
\hline \multirow[b]{2}{*}{ Facteurs expérimentaux } & \multicolumn{4}{|c|}{ Variables observées } \\
\hline & $\begin{array}{c}\text { PPG } \\
\text { (\% de tubercules sains) }\end{array}$ & $\mathbf{P P}(\%)$ & Bo $(\%)$ & $\mathrm{Pa}(\%)$ \\
\hline \multicolumn{5}{|l|}{ Variété } \\
\hline Rouge & - & $32,48 \mathrm{a}$ & $0,36 \mathrm{a}$ & $34,18 \mathrm{a}$ \\
\hline Blanche & - & $25,33 b$ & $8,05 b$ & $39,93 b$ \\
\hline \multicolumn{5}{|l|}{ Stade de maturité } \\
\hline 8 mois & $0.00 \mathrm{a}$ & $40,35 \mathrm{a}$ & $3,67 \mathrm{a}$ & $7,14 \mathrm{a}$ \\
\hline 9 mois & $0.00 \mathrm{a}$ & $38.55 b$ & $3,46 a$ & $24,80 \mathrm{~b}$ \\
\hline 10 mois & $31,90 b$ & $29,49 c$ & $4,69 \mathrm{ab}$ & $56,44 \mathrm{c}$ \\
\hline 11 mois & $33,90 b$ & $28,91 \mathrm{c}$ & $5,03 \mathrm{~b}$ & $59,83 \mathrm{c}$ \\
\hline \multicolumn{5}{|l|}{ Chloration } \\
\hline $0 \% \mathrm{NaOCl}$ & $59,89 a$ & $27,29 a$ & - & - \\
\hline $1 \% \mathrm{NaOCl}$ & $72,19 b$ & $34,54 \mathrm{~b}$ & - & - \\
\hline \multicolumn{5}{|l|}{ Emballage } \\
\hline Sans emballage & - & $29,89 a$ & - & - \\
\hline Sacs en jute & - & $30,65 a$ & - & - \\
\hline Plastique & - & $25,90 \mathrm{~b}$ & - & - \\
\hline
\end{tabular}

Une amélioration significative $(\mathrm{p}<0,05)$ était enregistrée sur du macabo traité avec l'hypochlorite de sodium à la dose de $1 \%$ et sur des tubercules récoltés à 10 ou 11 mois, indépendamment de la variété (Tableau 1). Le traitement à l'eau chlorée a été inefficace à empêcher la pourriture des tubercules emballés dans des sacs en jute. Quant à ceux entreposés dans des sacs en plastique, et dans une moindre mesure ceux des sacs en plastique perforé, ils ont développé une pourriture rapide, nettement accentuée quand le trempage dans la solution chlorée était effectué avant l'emballage (Tableau 1).

Les sacs en plastique perforé ont de façon significative $(\mathrm{p}<0,05)$ réduit l'envergure de la perte d'eau sur les tubercules entreposés. Une perte de poids remarquable était par contre enregistrée sur les tubercules non emballés et ceux dans des sacs en jute (Tableau 1).

Avec le temps d'avancement de la période d'entreposage, une chute de près de la moitié de la valeur initiale de la teneur en eau a été enregistrée sur le macabo rouge (Figure
1) et de manière plus marquée sur le macabo blanc. La chloration s'est révélée sans effet sur la teneur en eau durant l'entreposage. Les 2 variétés comportaient presque le même taux élevé de perte d'eau lorsque la récolte s'est effectuée au stade immature (Figure 2) ou en l'absence d'emballage (Figure 3).

La qualité organoleptique du macabo cuit, au terme de la période d'entreposage, a varié de manière significative $(p<0,05)$ suivant la variété du produit et beaucoup plus selon le stade de maturité des tubercules. Le macabo blanc était mieux apprécié (Tableau 2).

\section{DISCUSSION}

La perte de poids élevée du macabo blanc résulte de l'intense activité physiologique, notamment le taux élevé de respiration de ce macabo, inhérente sans doute à une caractéristique génétique de cette variété, et du stade sans doute plus précoce de récolte des tubercules de ce macabo. Une perte de poids remarquable était enregistrée sur les tubercules récoltés au stade de maturité 


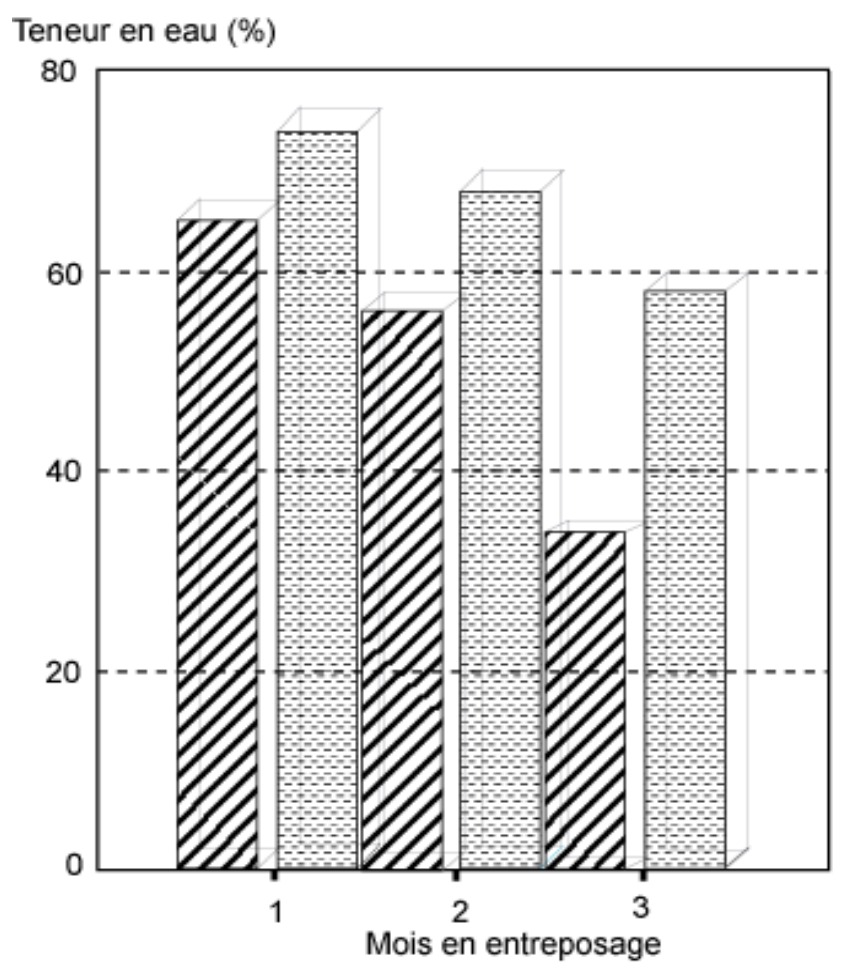

Légende: Variété de macabo
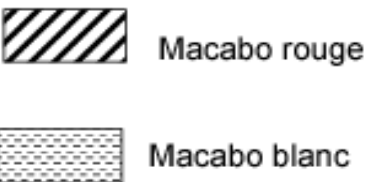

Figure 1 : Influence de la variété sur la teneur en eau du macabo entreposé.

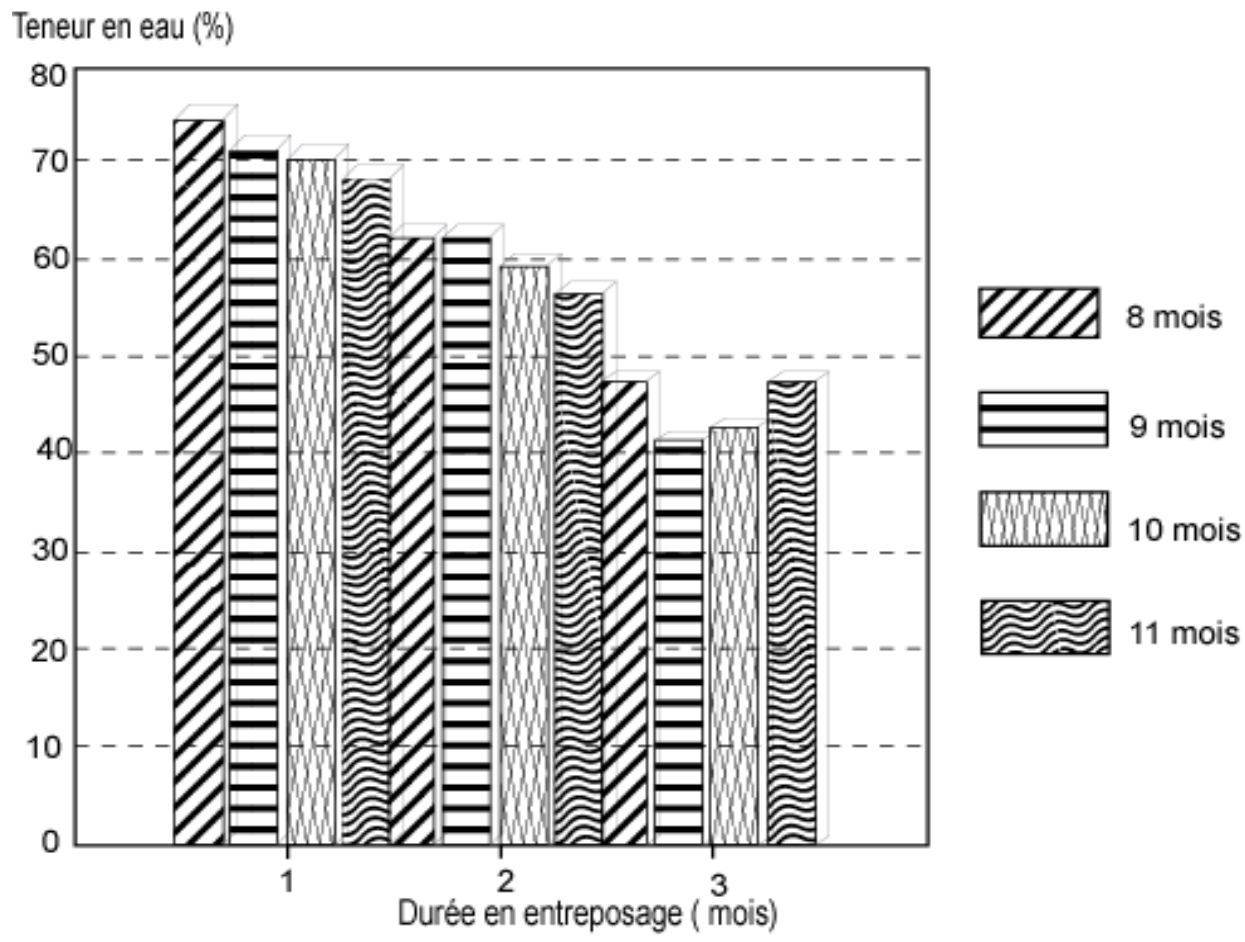

Figure 2 : Influence de la maturité sur la teneur en eau moyenne du macabo entreposé. 

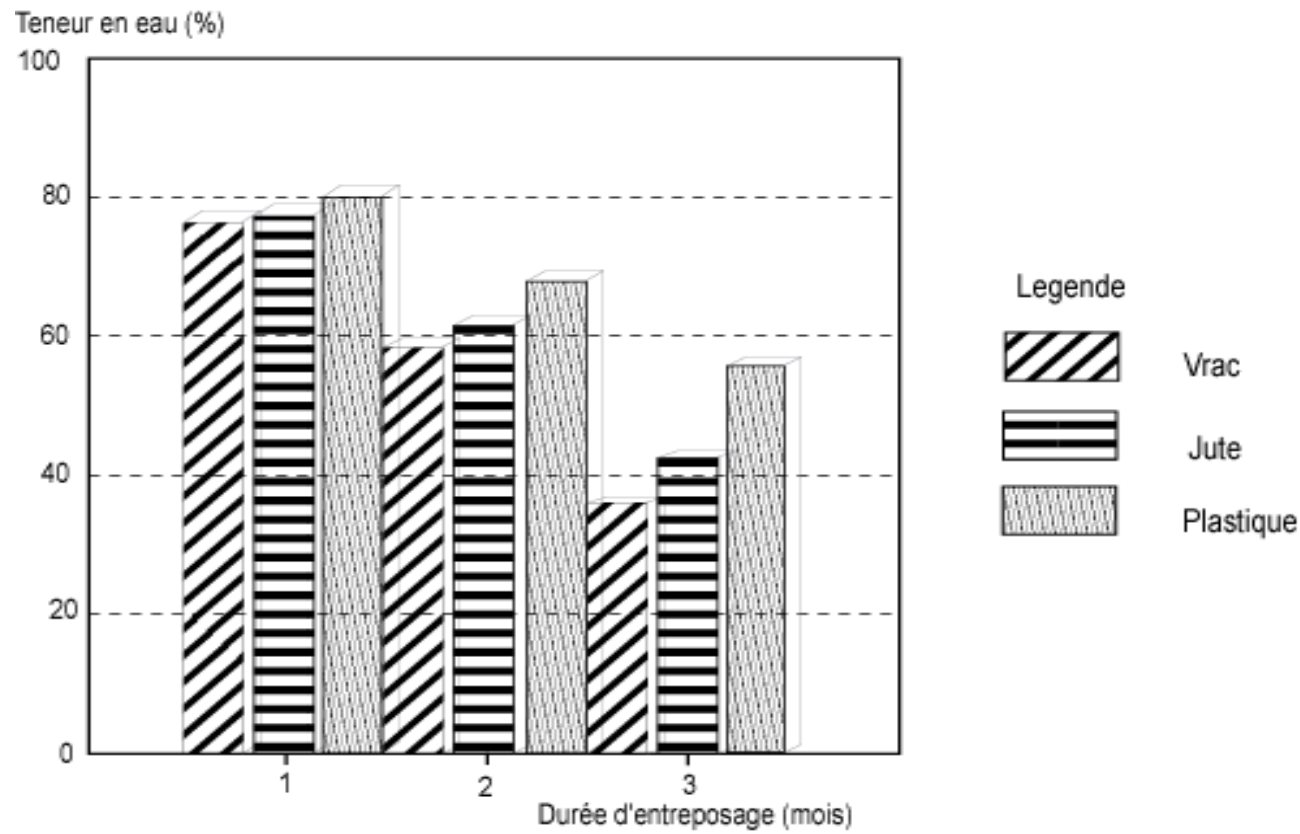

Figure 3 : Influence du type d'emballage sur la teneur en eau moyenne du macabo entreposé.

de 8 à 9 mois, supérieur au niveau obtenu sur ceux récoltés à 10 et 11 mois, en rapport sans doute avec la respiration et la transpiration intensives ayant normalement cours dans les tissus végétaux relativement immatures (Ora, 1977).

Le développement abondant de bourgeons sur le macabo blanc serait imputable à la maturation physiologique rapide, à l'absence de stimulation effective des substances inhibitrices de croissance, ou à l'absence de concentration optimale de cellesci (Isenberg et al., 1987). Le contrôle de la dormance du macabo blanc dans les conditions expérimentales de notre étude s'avère par conséquent hypothétique et incertain. Cet handicap se rencontre dans l'entreposage des tubercules d'aracée (Obetta et al., 2007). Le bourgeonnement était par ailleurs moins manifesté sur des tubercules récoltés à 8 à 9 mois que sur ceux âgés de 10 à 11 mois, autant pour le macabo rouge que pour le macabo blanc. Il est de ce fait confirmé que le stade physiologique immature constitue une cause naturelle de l'inaptitude de bourgeonnement (Burton, 1982).

La pourriture des tubercules de macabo emballés dans des sacs en plastique était sans doute causée par l'humidité persistante sur les tubercules après un séchage incomplet de ceux-ci, suivant leur trempage à l'eau chlorée (Bartz et Kelman, 1985). La présence de fragments de terre résiduels et de débris de substrat de récolte sur des tubercules non nettoyés avant trempage était très susceptible d'atténuer, voire bloquer l'action bactéricide du chlore, à cause de la promptitude de combinaison de celui-ci avec ces débris telluriques ou végétaux ou tout autre matière organique présents dans la solution chlorée de trempage. La proportion de chlore libre et susceptible d'éradiquer des agents pathologiques se trouve de ce fait réduite (Brown et Wardowski, 1984; Bikomo et Brecht, 1991).

Par ailleurs, bien que l'hypochlorite de sodium ait pu neutraliser l'intégralité ou en partie les agents pathogènes sur la surface des tubercules, ceux déjà infiltrés dans des entailles et de lenticelles pourraient ne pas avoir subi ce traitement et seraient à mesure de résister énergiquement à l'effet du chlore sur leur croissance. De plus, leur multiplication et leur agressivité ont été relancées et ont pris d'ampleur après que l'action du chlore se soit dissipée et que des conditions ambiantes favorables soient revenues. Afin de contrecarrer ce revirement, il est recommandable d'accroître le temps de trempage de 2 à 5 minutes, pour améliorer 
l'efficacité du chlore dans cette méthode; de même, l'augmentation adéquate de la concentration de chlore et de la modification du $\mathrm{pH}$ pourraient renforcer l'effet bactéride de la solution de trempage (Segall, 1968; Bikomo et Brecht, 1991)

En plus du fait que la perte d'eau soit accélérée sur des produits exposés à l'air, l'absorption de l'eau des tubercules dans les sacs en jute était particulièrement accentuée par la proximité et le contact avec ce type d'emballage, confirmant les résultats d'une étude précédente sur l'entreposage du macabo (Bikomo, 1988; Bikomo et Brecht, 1991). Les emballages en plastique perforé ont favorisé le développement d'un niveau d'humidité relative adéquat et aménagé une ventilation convenable ayant certainement contribué à minimiser la perte d'eau.

La réduction progressive de la teneur en eau dans les tubercules (Figures 2 et 3 ) s'accompagnait d'une augmentation considérable de la matière sèche. La diminution très rapide de la teneur en eau des produits récoltés au stade immature était imputable à la présence d'un périderme externe mince et plus perméable à la vapeur d'eau, en comparaison à celui plus épais et moins sujet au transfert de l'eau (Booth et Shaw, 1981; Wills et al., 1981; Burton, 1982; Ben-Yehoshua, 1987). Le macabo rouge semble présenter une enveloppe comportant ces caractéristiques favorables au transfert d'eau, contrairement au macabo blanc. La diminution rapide vers un seuil minimum critique de la teneur en eau des tubercules de macabo (Figure 3), et spécifiquement ceux emballés dans des sacs en jute proviendrait sans doute également de l'influence de la capacité élevée d'absorption d'eau et le caractère desséchant du jute, utilisé comme matière d'emballage ou de l'atmosphère environnante.

La préférence du macabo blanc tenait plus sur la palatabilité des tubercules de cette variété, ayant été préservée, après entreposage, assez identique à celui des produits fraîchement récoltés. En fait, le macabo blanc ramollit relativement vite et davantage à l'opposé du macabo rouge, dans les mêmes conditions de cuisson. Par ailleurs, la flaveur des tubercules récoltés après 10 à 11 mois était plus appréciée. Ceux récoltés à un stade de maturité précoce étaient insipides et atteignaient un degré de ramollissement et d'inhibition d'eau les rendant indésirables et impropres à la consommation. L'emballage et la chloration n'ont pas eu d'incidence sur la palatabilité du macabo entreposé.

\section{REMERCIEMENTS}

Le financement des travaux de ce projet de recherche a été assuré par la Fondation Internationale pour la Science (FIS).

\section{BIBLIOGRAPHIE}

Bainbridge K, Tomlins K, Wellings K, Westby A. 1996. Methods for Assessing Quality Characteristics of Non-grain Starch Staples (Part 3. Laboratory Methods). Natural Resources Institute: Chatham, UK.

Bart JA, Kelman A. 1985. Effect of air-drying of soft rot potential of potato tubers inoculated by immersion in suspension of Erwinia carotovora. Plant Disease, 69(2): 128-131.

Ben Yehoshua S. 1987. Individual sealpackaging of fruit and vegetables in plastic film. A new postharvest technique. Hort Science, 20(1): 32-37.

Bikomo MR. 1988. Postharvest handling and storage of Xanthosoma (Xanthosoma spp). M. Sc. Thesis. University of Florida, Grainesville, USA, 153p.

Bikomo MR, Brecht JK. 1991. Curing, wash water chlorination and packaging to improve the postharvest quality of Xanthosoma cormels. Scientia Horticulturae, 47: 1-13.

Booth RH. 1974. Post harvest deterioration of tropical root crops: losses and their control. Trop. Sc., 16(2): 49-63.

Booth RH, Shaw RL. 1981. Principles of Potato Storage. International Potato Center: Lima, Peru; 105p.

Brown GE, Wardoyski WF. 1984. Use of chlorine and chlorine dioxide in Florida citrus packing-houses to reduce inoculum of decay pathogens. Proc. Flo. State Hort. Soc., 97: 97-100.

Burton WG. 1982. Postharvest Physiology of Food Crops. Longman: London and New-York.

Coursey DG. 1983. Post harvest losses in perishable foods of the developing world. In Post Harvest Physiology and Crop Preservation, Lieberman M (ed). Plenum Press: New-York and London; 492-493. 
Delate K, Brecht JK, Lambert M. 1985. Impact of curing, film wrapping and chlorination on the storability of Boniato sweet potato. Proc. Fla. State Hort. Soc., 98: $187-198$.

Emmanuel-Ipkeme CA, Eneji CA, Essiet U. 2007. Storage stability and sensory evaluation of taro chips fried in palm oil, palm olein oil, groundnut oil, soybean oil and their blends. Pakistan Journal of Nutrition, 6(6): 570-575.

Isenberg FMR, Thomas TH, Ludford PM. 1987. Hormonal alterations during the postharvest. In Posharvest Physiology of Vegetables, Weichmann J (ed). Marcel Dekker Inc.: New York and Basel; 45-94.

Kader AA, Lipton WJ, Morris LL. 1973. Systems for scoring quality of harvested lettuce. Hort Science, 8(5): 408-409.

Larmond E. 1977. Laboratory Methods for Sensory Evaluation of Food. Canada Department of Agriculture, publication 1637: Ottawa; 73p.

Nzietchueng S. 1983. La pourriture racinaire du macabo (Xanthosoma sagittifolium) au Cameroun: 1. Symptomatologie et étiologie de la maladie. Agronomia Tropical, 38(4) : 321-325.

Nzietchueng S. 1984. La pourriture racinaire de macabo (Xanthosoma sagittifolium) au Cameroun: 1. Symptomatologie et étiologie de la maladie. Agron. Trop., 38(4): 321-325.

Obetta SE, Ijabo OJ, Satimehin AA. 2007. Evaluation of a ventilated underground storage for cocoyams (taro). Agricultural Engineering International: the CIGR Journal, 9: 13.

Ogundana SK. 1977. The pathogenicity of the fungi causing rot in the corms of cocoyam (Xanthosoma sagittifolium). Inter. Biodeterioration Bulletin, 13(1): 58.

O’Hair SK, Asokan MP. 1986. Edible aroids. Botany and horticulture. Horticultural Reviews, 8: 43-49,

Ora S. 1977. Potatoes: Production, Storing, Processing. Avi Publishing Company Inc.: Westport, Conn.

Passam HC. 1982b. Experiments on the storage of eddoes and tannins (Colocassia and Xanthosoma) under tropical ambient conditions. Tropical Sciences, 24: 39-46.

Rickard JE, Coursey DG. 1981. Cassava storage. Part 1, Storage of fresh cassava root. Trop. Sc., 23(1): 1-32.

Segall RH. 1968. Fungicidal effectiveness of chlorine as influenced by concentration, temperature, $\mathrm{pH}$ and spore exposure time. Phytopathology, 58: 1412-1414.

Shillinford CA. 1978. Effect of plastic coating and polyethylene wraps on banana fruit rot and quality. Trop. Agr., 55(2): 173179.

Tiky MG. 1998. Cassava and soyabean flours and resting time effects on sensory and physical characteristics of reconstituted miond. J. Sci. Food Agric., 76: 310-314.

Watts BM, Ylimaki GL, Jeffery LE, Elias LG. 1991. Methodes de Base pour l'Evaluation Sensorielle des Aliments. Centre de Recherches pour le Développement International (CRDI): Ottawa, Ontario, Canada; 145 p.

Wills RBH, Lee TH, Graham D, McGlasson WB, Hall EG. 1981. Postharvest. An Introduction to the Physiology and Handling of Fruits and Vegetables. Avi Publishing Company Inc.: Westport, Conn. 